\section{The Structures of Pyridovericin and Pyridomacrolidin, New Metabolites from the Entomopathogenic Fungus, Beauveria bassiana}

\author{
Senji Takahashi, Kenichi Uchida, \\ Norihiro Kakinuma ${ }^{\dagger}$, Ryuju Hashimoto ${ }^{\dagger \dagger}$, \\ Tadashi Yanagisawa ${ }^{\dagger}$ and Akira Nakagawa* \\ Department of Biosciences, Teikyo University, \\ 1-1 Toyosatodai, Utsunomiya 320-8551, Japan \\ ${ }^{\dagger}$ Faculty of Agriculture, Utsunomiya University, \\ 350 Mine-machi, Utsunomiya 321-8505, Japan
}

(Received for publication August 10, 1998)

Entomopathogenic fungi seem to be interesting as a source of biologically active compounds such as an insecticides $^{1)}$ and immunosuppressants ${ }^{2,3)}$, because all insect orders are susceptible to fungal diseases. With the goal of finding novel bioactive compounds from fungi, we investigated metabolite diversity using an HPLC screening program. During the screening process, we isolated two novel metabolites named pyridovericin (1) and pyridomacrolidin (2) (Fig. 1) from the mycelial cake of the entomopathogenic fungus, Beauveria bassiana EPF- $5^{4}$. In this paper, we describe the physico-chemical properties and structural determination of $\mathbf{1}$ and $\mathbf{2}$.

\section{Structure of Pyridovericin (1)}

The physico-chemical properties of $\mathbf{1}$ and $\mathbf{2}$ are summarized in Table 1. The molecular formula of $\mathbf{1}$ was determined to be $\mathrm{C}_{21} \mathrm{H}_{23} \mathrm{NO}_{5}$ [Found $m / z \quad 369.1580$ $\left(\mathrm{M}^{+}\right)$, Calcd for $\mathrm{C}_{21} \mathrm{H}_{23} \mathrm{NO}_{5}$ 369.1576] by highresolution EI-MS measurement. The IR spectrum $(\mathrm{KBr})$ suggested the presence of the hydroxy group $\left(3403 \mathrm{~cm}^{-1}\right)$, amide carbonyl $\left(1661 \mathrm{~cm}^{-1}\right)$ and hydrogen bonded carbonyl $\left(1611 \mathrm{~cm}^{-1}\right)$, respectively.

The ${ }^{13} \mathrm{C}$ NMR spectrum of $\mathbf{1}$ accounts for the presence of 21 carbon signals. The analyses of ${ }^{1} \mathrm{H}$ NMR and DEPT spectra indicated the presence of two methyls $\left(\delta_{\mathrm{C}}\right.$ 11.6 and 12.7), one methylene $\left(\delta_{C} 24.0\right)$, one oxygenated methylene $\left(\delta_{\mathrm{C}} 64.0\right)$, one methine $\left(\delta_{\mathrm{C}} 43.5\right)$, eight olefinic methines $\left(\delta_{\mathrm{C}} 114.9 \times 2,123.1,130.0 \times 2,140.5,147.4\right.$ and 149.3) and eight quaternary carbons including six olefinic carbons $\left(\delta_{\mathrm{C}} 105.9,112.7,123.4,134.5,156.7\right.$ and 176.9) and two carbonyl carbons $\left(\delta_{\mathrm{C}} 161.7\right.$ and 193.7). The ${ }^{1} \mathrm{H}$ NMR spectrum showed 23 proton signals including two methyls $\left(\delta_{\mathrm{H}} 0.82, \mathrm{t}\right.$ and $\left.\delta_{\mathrm{H}} 1.85, \mathrm{~s}\right)$, one methylene $\left(\delta_{\mathrm{H}}\right.$ $1.22, \mathrm{~m}$ and $\left.\delta_{\mathrm{H}} 1.59, \mathrm{~m}\right)$, one oxygenated methylene $\left(\delta_{\mathrm{H}}\right.$ $3.37,2 \mathrm{H}, \mathrm{m})$, one methine $\left(\delta_{\mathrm{H}} 2.53, \mathrm{~m}\right)$, three olefinic protons $\left(\delta_{\mathrm{H}} 5.95, \mathrm{~d}, \delta_{\mathrm{H}} 7.51, \mathrm{~d}\right.$ and $\left.\delta_{\mathrm{H}} 8.00, \mathrm{~d}\right)$, one aromatic proton $\left(\delta_{\mathrm{H}} 7.54, \mathrm{~s}\right)$ and $p$-disubstituted benzene protons $\left(\delta_{\mathrm{H}} 6.78,2 \mathrm{H}\right.$, d and $\left.\delta_{\mathrm{H}} 7.27,2 \mathrm{H}, \mathrm{d}\right)$. Furthermore, the existence of four exchangeable protons: one aliphatic hydroxy proton $\left(\delta_{\mathrm{H}} 4.56, \mathrm{t}\right)$, one phenolic hydroxy proton $\left(\delta_{\mathrm{H}} 9.45, \mathrm{~s}\right)$, one $\mathrm{NH}$ proton $\left(\delta_{\mathrm{H}} 11.58\right.$, br.s $)$ and one hydrogen bonded phenolic hydroxy proton $\left(\delta_{\mathrm{H}} 17.54\right.$, br.s) was demonstrated. The chemical shift data of ${ }^{1} \mathrm{H}$ and ${ }^{13} \mathrm{C}$ NMR (DMSO- $d_{6}$ ) of $\mathbf{1}$ are shown in Table 2.

The ${ }^{1} \mathrm{H}^{-}{ }^{1} \mathrm{H}$ COSY, ${ }^{1} \mathrm{H}^{-1}{ }^{13} \mathrm{C}$ COSY and $\mathrm{HMBC}$ spectra of 1 revealed the presence of two partial structures $A$ and $\mathrm{B}$, a 4-hydroxy-5-( $p$-hydroxyphenyl) pyridone moiety and a 4-methyl-6-hydroxymethyl-octa-2,4-dienoyl moiety, respectively, as shown in Fig. 2. The presence of the $p$-hydroxyphenyl moiety deduced from the appearance of proton signals of typical $\mathrm{AA}^{\prime} \mathbf{B B}^{\prime}$ multiplets and two carbon signals arising from the chemically equivalent pairs of carbons $2^{\prime}-\mathrm{C}, 6^{\prime}-\mathrm{C}$ and $3^{\prime}-\mathrm{C}, 5^{\prime}-\mathrm{C}$ was evidenced by the correlations from $4^{\prime}-\mathrm{OH}$ $\left(\delta_{\mathrm{H}} 9.45\right)$ to $4^{\prime}-\mathrm{C}\left(\delta_{\mathrm{C}} 156.7\right)$ and $3^{\prime}\left(\right.$ or $\left.5^{\prime}\right)-\mathrm{C}\left(\delta_{\mathrm{C}} 114.9\right)$, from $3^{\prime}$ (or $\left.5^{\prime}\right)-\mathrm{H}\left(\delta_{\mathrm{H}} 6.78\right)$ to $4^{\prime}-\mathrm{C}$, and from $2^{\prime}\left(\right.$ or $\left.6^{\prime}\right)-\mathrm{H}$

Fig. 1. Structures of pyridovericin (1) and pyridomacrolidin (2).

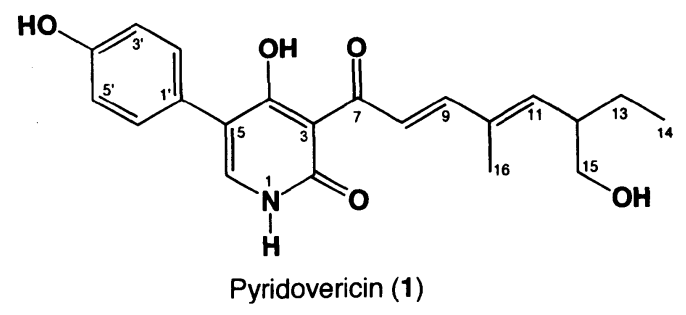

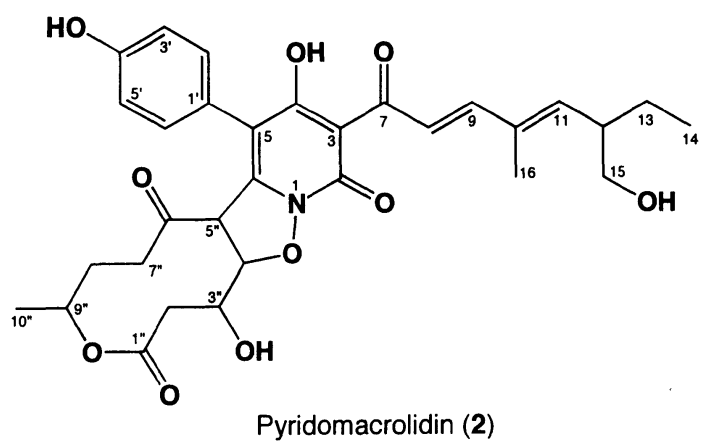

${ }^{+\dagger}$ Present address: Kissei Pharmaceutical Co. Ltd., 3-1-3 Koishikawa, Bunkyo-ku, Tokyo 112-0002, Japan. 
Table 1. Physico-chemical properties of pyridovericin (1) and pyridomacrolidin (2).

\begin{tabular}{|c|c|c|}
\hline & Pyridovericin (1) & Pyridomacrolidin (2) \\
\hline Appearance & Pale yellow powder & Pale yellow powder \\
\hline $\begin{array}{l}\mathrm{MP} \\
{[\alpha]_{\mathrm{D}}^{15}(c 0.1, \mathrm{MeOH})}\end{array}$ & $\begin{array}{l}203 \sim 206{ }^{\circ} \mathrm{C}(\text { dec. }) \\
-20.3^{\circ}\end{array}$ & $\begin{array}{l}192 \sim 194^{\circ} \mathrm{C}(\text { dec. }) \\
+19.4^{\circ}\end{array}$ \\
\hline Molecular formula & $\mathrm{C}_{21} \mathrm{H}_{23} \mathrm{NO}_{5}$ & $\mathrm{C}_{31} \mathrm{H}_{35} \mathrm{NO}_{10}$ \\
\hline Molecular weight & 369 & 581 \\
\hline FAB-MS $(m / z)$ & $\begin{array}{l}370(\mathrm{M}+\mathrm{H})^{+} \\
368(\mathrm{M}-\mathrm{H})^{-}\end{array}$ & $\begin{array}{l}582(\mathrm{M}+\mathrm{H})^{+} \\
580(\mathrm{M}-\mathrm{H})^{-}\end{array}$ \\
\hline HR-MS $(m / z)$ & EI & FAB \\
\hline Found: & $369.1580(\mathrm{M})^{+}$ & $582.2329(\mathrm{M}+\mathrm{H})^{+}$ \\
\hline Calcd: & 369.1576 for $\mathrm{C}_{21} \mathrm{H}_{23} \mathrm{NO}_{5}$ & 582.2339 for $\mathrm{C}_{3}{ }_{1} \mathrm{H}_{3} 6 \mathrm{NO}_{10}$ \\
\hline $\mathrm{UV} \lambda_{\max }^{\mathrm{MeOH}} \mathrm{nm}(\varepsilon)$ & $248(16,400), 336(15,900)$ & $238(14,500), 342(14,600)$ \\
\hline $\operatorname{IR} v_{\max }^{\mathrm{KBr}}\left(\mathrm{cm}^{-1}\right)$ & $3403,2963,2934,1661,1611,1578$ & $3399,2961,2930,1723,1643,1613$ \\
\hline & $\begin{array}{l}1522,1474,1462,1325,1258,1223 \\
1034,992\end{array}$ & $\begin{array}{l}1518,1441,1433,1400,1343,1267 \\
1227,1173,1092,1038,984\end{array}$ \\
\hline TLC (Rf value) ${ }^{a}$ & 0.34 & 0.44 \\
\hline $\begin{array}{ll}\text { Solubility } & \text { Soluble: } \\
& \text { Insoluble: }\end{array}$ & $\begin{array}{l}\mathrm{MeOH}, \text { EtOAc, DMSO, acetone } \\
\mathrm{H}_{2} \mathrm{O} \text {, n-hexane }\end{array}$ & $\begin{array}{l}\mathrm{MeOH}, \text { EtOAc, DMSO, acetone, } \mathrm{CHCl}_{3} \\
\mathrm{H}_{2} \mathrm{O} \text {, n-hexane }\end{array}$ \\
\hline
\end{tabular}

a Silica gel TLC (Merck Art. 5715): $\mathrm{CHCl}_{3}-\mathrm{MeOH}(8: 1)$.

$\left(\delta_{\mathrm{H}} 7.27\right)$ to $4^{\prime}-\mathrm{C}$ and $5-\mathrm{C}\left(\delta_{\mathrm{C}} 112.7\right)$. The location at 4-OH $\left(\delta_{\mathrm{H}} 17.54\right)$ was assigned from the correlation to three carbons, 4-C $\left(\delta_{\mathrm{C}} 176.9\right), 3-\mathrm{C}\left(\delta_{\mathrm{C}} 105.9\right)$ and 5-C $\left(\delta_{\mathrm{C}}\right.$ 112.7). Furthermore, the appearance of correlations of a singlet olefinic proton $6-\mathrm{H}\left(\delta_{\mathrm{H}} 7.54\right)$ to an amide carbonyl 2-C ( $\delta_{\mathrm{C}}$ 161.7), 4-C, 5-C and 1'-C ( $\left.\delta_{\mathrm{C}} 123.4\right)$ indicated the presence of a pyridone ring in partial structure A. The linkage of $p$-hydroxyphenyl group to the 5-C position of pyridone moiety was established by correlations from $2^{\prime}$ (or $6^{\prime}$ )- $\mathrm{H}$ to $5-\mathrm{C}$ and from $6-\mathrm{H}$ to $1^{\prime}-\mathrm{C}$, as shown in Fig. 2. The ${ }^{1} \mathrm{H}^{-1} \mathrm{H}$ COSY spectrum of 1 revealed two proton sequences $\left[-{ }^{8} \mathrm{CH}={ }^{9} \mathrm{CH}-\right.$ and $\left.{ }^{11} \mathrm{CH}-{ }^{12} \mathrm{CH}\left({ }^{15} \mathrm{CH}_{2} \mathrm{OH}\right)-{ }^{13} \mathrm{CH}_{2}-{ }^{14} \mathrm{CH}_{3}\right]$ in partial structure $\mathrm{B}$. In the $\mathrm{HMBC}$ spectrum, a methyl proton 16- $\mathrm{CH}_{3}\left(\delta_{\mathrm{H}} 1.85\right)$ showed correlations with three olefinic carbons $9-\mathrm{C}\left(\delta_{\mathrm{C}} 149.3\right), 10-\mathrm{C}\left(\delta_{\mathrm{C}} 134.5\right)$ and 11-C $\left(\delta_{\mathrm{C}}\right.$ 147.4). Both olefinic protons, $8-\mathrm{H}\left(\delta_{\mathrm{H}} 8.00\right)$ and $9-\mathrm{H}\left(\delta_{\mathrm{H}}\right.$ 7.51) were correlated to a carbonyl carbon 7-C $\left(\delta_{\mathrm{C}} 193.7\right)$. The terminal olefinic methine proton $11-\mathrm{H}\left(\delta_{\mathrm{H}} 5.95\right)$ was correlated to an olefinic carbons $10-\mathrm{C}$ and $9-\mathrm{C}$. The configuration of the two double bonds (8-C to $9-\mathrm{C}$ and 10-C to 11-C) were elucidated as $E$ from its coupling constant $\left(J_{8,9}=15.6 \mathrm{~Hz}\right)$ and observation of NOE between $9-\mathrm{H}$ and $11-\mathrm{H}$, and $8-\mathrm{H}$ and $16-\mathrm{CH}_{3}$, respectively. These data deduced $(2 E, 4 E)$-4-methyl-6-hydroxymethylocta-2,4-dienoyl moiety for the partial structure B. The connectivity of the carbonyl carbon $7-\mathrm{C}$ to $3-\mathrm{C}$ on a pyridone ring was deduced from the fact that a phenolic 4-OH $\left(\delta_{\mathrm{H}} 17.54\right)$ should be hydrogen bonded to the carbonyl oxygen (IR; $1611 \mathrm{~cm}^{-1}$ ). Thus, the planner structure of 1 was determined to be a 3-[(2E,4E)-4methyl-6-hydroxymethyl-octa-2,4-dienoyl]-4-hydroxy5-( $p$-hydroxyphenyl)-2(1H) pyridone, as shown in Fig. 1.

\section{Structure of Pyridomacrolidin (2)}

The molecular formula of $\mathbf{2}$ was established to be $\mathrm{C}_{31} \mathrm{H}_{35} \mathrm{NO}_{10}$ by high-resolution FAB-MS [Found $\mathrm{m} / \mathrm{z}$ $582.2329(\mathrm{M}+\mathrm{H})^{+}$, calcd for $\mathrm{C}_{31} \mathrm{H}_{36} \mathrm{NO}_{10}$ 582.2339]. The UV spectrum of $\mathbf{2}$ in $\mathrm{MeOH}$ exhibited an absorption maxima at $238 \mathrm{~nm}(\varepsilon 14,500)$ and $342 \mathrm{~nm}(\varepsilon 14,600)$, suggesting that the compound contained the same chromophore with 1. The IR spectrum of 2 showed a newly appeared absorption of ester carbonyl $\left(1723 \mathrm{~cm}^{-1}\right)$ in addition to a hydroxy group $\left(3399 \mathrm{~cm}^{-1}\right)$, amide carbonyl $\left(1643 \mathrm{~cm}^{-1}\right)$ and hydrogen bonded carbonyl $\left(1613 \mathrm{~cm}^{-1}\right)$.

Comparative studies of ${ }^{1} \mathrm{H}$ and ${ }^{13} \mathrm{C}$ NMR spectral data (see Table 2) of $\mathbf{1}$ and $\mathbf{2}$ indicated that $\mathbf{2}$ possesses the same structure as $\mathbf{1}$, except for the signals of an amide proton and olefinic proton at $6-\mathrm{C}$ observed in $\mathbf{1}$, as shown in Fig. 3. The $E$-configuration of two double bonds, 8-C 
Table 2. ${ }^{1} \mathrm{H}$ and ${ }^{13} \mathrm{C}$ NMR chemical shift assignments of pyridovericin (1) and pyridomacrolidin (2) ${ }^{\mathrm{a}}$.

\begin{tabular}{|c|c|c|c|c|}
\hline \multirow[b]{2}{*}{ Position } & \multicolumn{2}{|c|}{${ }^{13} \mathrm{C}(\delta)$} & \multicolumn{2}{|c|}{${ }^{1} \mathrm{H}(\delta)$} \\
\hline & 1 & 2 & 1 & 2 \\
\hline $\mathrm{NH}-1$ & & & $11.58(1 \mathrm{H}, \mathrm{br} . \mathrm{s})^{\mathrm{b}}$ & \\
\hline$C-2$ & 161.7 & 153.1 & & \\
\hline$C-3$ & 105.9 & 105.6 & & \\
\hline C-4 & 176.9 & 173.7 & & \\
\hline $\mathrm{C}-4-\mathrm{OH}$ & & & $17.54(1 \mathrm{H}$, br.s $)$ & $16.71(1 \mathrm{H}, \mathrm{s})$ \\
\hline C-5 & 112.7 & 106.5 & & \\
\hline C-6 & 140.5 & 145.0 & $7.54(1 \mathrm{H}, \mathrm{s})$ & \\
\hline $\mathrm{C}-7$ & 193.7 & 193.1 & & \\
\hline$C-8$ & 123.1 & 122.6 & $8.00(1 \mathrm{H}, \mathrm{d}, J=15.6 \mathrm{~Hz})$ & $7.90(1 \mathrm{H}, \mathrm{d}, J=15.1 \mathrm{~Hz})$ \\
\hline C-9 & 149.3 & 149.9 & $7.51(1 \mathrm{H}, \mathrm{d}, J=15.6 \mathrm{~Hz})$ & $7.53(1 \mathrm{H}, \mathrm{d}, J=15.1 \mathrm{~Hz})$ \\
\hline $\mathrm{C}-10$ & 134.5 & 134.4 & & \\
\hline $\mathrm{C}-11$ & 147.4 & 148.1 & $5.95(1 \mathrm{H}, \mathrm{d}, J=9.8 \mathrm{~Hz})$ & $5.98(1 \mathrm{H}, \mathrm{d}, J=10.3 \mathrm{~Hz})$ \\
\hline$C-12$ & 43.5 & 43.6 & $2.53(1 \mathrm{H}, \mathrm{m})$ & $2.54(1 \mathrm{H}, \mathrm{m})$ \\
\hline \multirow[t]{2}{*}{$C-13$} & 24.0 & 23.9 & $1.22(1 \mathrm{H}, \mathrm{m})$ & $1.24(1 \mathrm{H}, \mathrm{m})$ \\
\hline & & & $1.59(1 \mathrm{H}, \mathrm{m})$ & $1.58(1 \mathrm{H}, \mathrm{m})$ \\
\hline$C-14$ & 11.6 & 11.6 & $0.82(3 \mathrm{H}, \mathrm{t}, J=7.6 \mathrm{~Hz})$ & $0.83(3 \mathrm{H}, \mathrm{t}, J=7.6 \mathrm{~Hz})$ \\
\hline$C-15$ & 64.0 & 64.0 & $3.37(2 \mathrm{H}, \mathrm{m})$ & $3.37(2 \mathrm{H}, \mathrm{m})$ \\
\hline \multicolumn{2}{|l|}{$\mathrm{C}-15-\mathrm{OH}$} & & $4.56(1 \mathrm{H}, \mathrm{t}, J=5.4 \mathrm{~Hz})$ & $4.56(1 \mathrm{H}, \mathrm{t}, J=5.4 \mathrm{~Hz})$ \\
\hline$C-16$ & 12.7 & 12.7 & $1.85(3 \mathrm{H}, \mathrm{s})$ & $1.86(3 \mathrm{H}, \mathrm{s})$ \\
\hline$C-1^{\prime}$ & 123.4 & 121.5 & & \\
\hline$C-2^{\prime}, 6^{\prime}$ & 130.0 & 131.6 & $7.27(2 \mathrm{H}, \mathrm{d}, J=8.3 \mathrm{~Hz})$ & $7.09(2 \mathrm{H}, \mathrm{d}, J=8.8 \mathrm{~Hz})$ \\
\hline$C-3,5^{\prime}$ & 114.9 & 115.7 & $6.78(2 \mathrm{H}, \mathrm{d}, J=8.3 \mathrm{~Hz})$ & $6.88(2 \mathrm{H}, \mathrm{d}, J=8.8 \mathrm{~Hz})$ \\
\hline C-4' & 156.7 & 157.7 & & \\
\hline $\mathrm{C}-4^{\prime}-\mathrm{OH}$ & & & $9.45(1 \mathrm{H}, \mathrm{s})$ & $9.72(1 \mathrm{H}, \mathrm{s})$ \\
\hline C-1" & & 168.4 & & \\
\hline \multirow[t]{2}{*}{ C-2" } & & 40.7 & & $2.47(1 \mathrm{H}, \mathrm{dd}, J=13.2,5.4 \mathrm{~Hz})$ \\
\hline & & & & $2.75(1 \mathrm{H}, \mathrm{dd}, J=13.2,2.2 \mathrm{~Hz})$ \\
\hline C-3" & & 67.5 & & $4.29(1 \mathrm{H}, \mathrm{m})$ \\
\hline C-3"-OH & & & & $6.06(1 \mathrm{H}, \mathrm{d}, J=4.4 \mathrm{~Hz})$ \\
\hline C-4" & & 87.8 & & $5.03(1 \mathrm{H}, \mathrm{d}, J=5.9 \mathrm{~Hz})$ \\
\hline C-5" & & 54.7 & & $5.23(1 \mathrm{H}, \mathrm{d}, J=5.9 \mathrm{~Hz})$ \\
\hline C-6" & & 207.4 & & \\
\hline C-7" & & 39.8 & & $1.36(1 \mathrm{H}, \mathrm{m}), 1.58(1 \mathrm{H}, \mathrm{m})$ \\
\hline C-8" & & 32.8 & & $1.58(1 \mathrm{H}, \mathrm{m}), 1.77(1 \mathrm{H}, \mathrm{m})$ \\
\hline C-9" & & 71.4 & & $4.71(1 \mathrm{H}, \mathrm{m})$ \\
\hline C-10" & & 18.5 & & $1.03(3 \mathrm{H}, \mathrm{d}, J=5.9 \mathrm{~Hz})$ \\
\hline
\end{tabular}

a ${ }^{1} \mathrm{H}$ NMR $(400 \mathrm{MHz})$ and ${ }^{13} \mathrm{C}$ NMR $(100 \mathrm{MHz})$ were measured in DMSO- $d_{6}$ at $30^{\circ} \mathrm{C}$.

b Proton number, multiplicity and coupling constants in $\mathrm{Hz}$ are indicated in parentheses.

and $10-\mathrm{C}$ were evidenced by NOE data $(9-\mathrm{H} / 11-\mathrm{H}$ and $8-\mathrm{H} / 16-\mathrm{CH} 3)$, and a coupling constant $\left(J_{8,9}=15.1 \mathrm{~Hz}\right)$. The remaining partial structure $\mathrm{C}\left(\mathrm{C}_{10} \mathrm{H}_{14} \mathrm{O}_{5}\right)$ in 2 was clarified from $\mathrm{HMBC}$ experiments among three proton sequences $\left[-{ }^{2}{ }^{\prime \prime} \mathrm{CH}_{2}-{ }^{3 \prime} \mathrm{CH}(\mathrm{OH})-,-\mathrm{O}-{ }^{4}{ }^{\prime \prime} \mathrm{CH}-{ }^{5 \prime} \mathrm{CH}-\right.$ and $\left.-{ }^{7 "} \mathrm{CH}_{2}-{ }^{8 \prime} \mathrm{CH}_{2}-{ }^{9 "} \mathrm{CH}\left({ }^{10 "} \mathrm{CH}_{3}\right)-\mathrm{O}-\right]$ assigned from the ${ }^{1} \mathrm{H}-{ }^{1} \mathrm{H}$ COSY spectrum. Observation of the correlations from $2^{\prime \prime}-\mathrm{H}_{2}\left(\delta_{\mathrm{H}} 2.47\right.$ and 2.75$)$ to $1^{\prime \prime}-\mathrm{C}\left(\delta_{\mathrm{C}}\right.$ $168.4)$, from $3^{\prime \prime}-\mathrm{H}\left(\delta_{\mathrm{H}} 4.29\right)$ to $4^{\prime \prime}-\mathrm{C}\left(\delta_{\mathrm{C}} 87.8\right)$, from $3^{\prime \prime}-\mathrm{OH}$ $\left(\delta_{\mathrm{H}} 6.06\right)$ to $4^{\prime \prime}-\mathrm{C}$, from $4^{\prime \prime}-\mathrm{H}\left(\delta_{\mathrm{H}} 5.03\right)$ to $2^{\prime \prime}-\mathrm{C}\left(\delta_{\mathrm{C}} 40.7\right)$, $3^{\prime \prime}-\mathrm{C}\left(\delta_{\mathrm{C}} 67.5\right)$ and $6^{\prime \prime}-\mathrm{C}\left(\delta_{\mathrm{C}} 207.4\right)$, from $5^{\prime \prime}-\mathrm{H}\left(\delta_{\mathrm{H}} 5.23\right)$ to $3^{\prime \prime}-\mathrm{C}$ and $6^{\prime \prime}-\mathrm{C}$, from $7^{\prime \prime}-\mathrm{H}_{2}\left(\delta_{\mathrm{H}} 1.36\right.$ and 1.58$)$ to $6^{\prime \prime}-\mathrm{C}$, from $8^{\prime \prime}-\mathrm{H}_{2}\left(\delta_{\mathrm{H}} 1.58\right.$ and 1.77$)$ to $6^{\prime \prime}-\mathrm{C}$, and from $9^{\prime \prime}-\mathrm{H}$ $\left(\delta_{\mathrm{H}} 4.71\right)$ to $1^{\prime \prime}-\mathrm{C}$ enabled the deduction of a 10 -membered macrocyclic structure for $\mathrm{C}$, as shown in Fig. 3. Although the oxygenated methine $4^{\prime \prime}-\mathrm{H}\left(\delta_{\mathrm{H}} 5.03, J_{4^{\prime \prime}, 5^{\prime \prime}}=5.9 \mathrm{~Hz}\right)$ showed no correlation with $3^{\prime \prime}-\mathrm{H}\left(\delta_{\mathrm{H}} 4.29\right)$, the observation of NOE between $3^{\prime \prime}-\mathrm{H}$ and $4^{\prime \prime}-\mathrm{H}$ suggests that the dihedral angle for both protons should be $c a$. $90^{\circ}$. The connectivity between partial structures $\mathrm{A}$ and $\mathrm{C}$ in $\mathbf{2}$ was determined from the following $\mathrm{HMBC}$ data. Appearance of the correlations from $5^{\prime \prime}-\mathrm{H}$ to $6-\mathrm{C}\left(\delta_{\mathrm{C}}\right.$ 
Fig. 2. ${ }^{1} \mathrm{H}^{-1} \mathrm{H}$ COSY and $\mathrm{HMBC}$ experiments of pyridovericin (1).

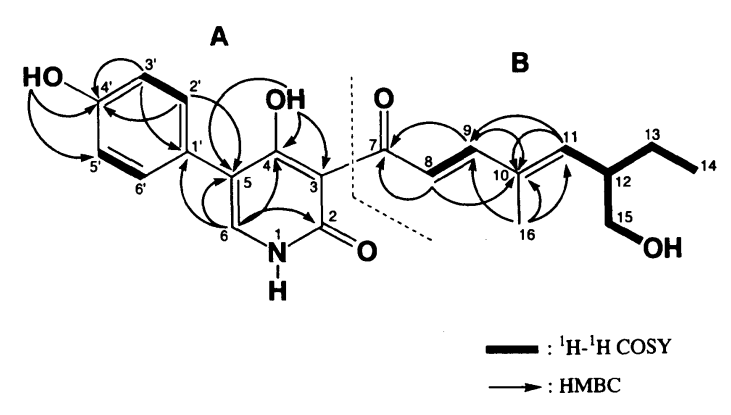

$145.0)$ and 5-C $\left(\delta_{\mathrm{C}} 106.5\right)$, and from $4^{\prime \prime}-\mathrm{H}$ to $6-\mathrm{C}$ indicated clearly that $5^{\prime \prime}-\mathrm{C}$ should be connected to $6-\mathrm{C}$ in the pyridone ring. Taking into consideration the abovedescribed $\mathrm{HMBC}$ data and degree of unsaturation, an oxygenated carbon $4^{\prime \prime}-\mathrm{C}\left(\delta_{\mathrm{C}} 87.8\right)$ should be connected to an amide nitrogen atom on the pyridone ring via an oxygen atom. Thus, compound 2 possesses an unique structure by which a 10 -membered macrocyclic lactone skeleton was linked to a pyridone ring of $\mathbf{1}$.

Although the $p$-hydroxyphenyl pyridone related compounds, tenellin, bassianin and ilicicolin $\mathrm{H}$, have been reported ${ }^{5,6)}$ from entomopathogenic fungi, Beauveria tenella, Beauveria bassiana and Cylindrocladium ilicicola, respectively, this is the first report of isolation of a novel pyridone compound with a 10-membered macrocyclic lactone.

\section{Experimental}

${ }^{1} \mathrm{H}$ NMR (400 MHz) and ${ }^{13} \mathrm{C}$ NMR $(100 \mathrm{MHz})$ spectra were measured on a JEOL JNM EX-400 spectrometer at $30^{\circ} \mathrm{C}$ in DMSO- $d_{6}$. FAB-MS spectrum was measured on a JEOL AX-505W mass spectrometer with a matrix of glycerol, 3-nitrobenzyl alcohol and polyethylenglycol. EI-MS spectrum was measured on a JEOL JMS-DX300 mass spectrometer. Infrared spectra $(\mathrm{KBr})$ and Ultraviolet spectra were taken on a Shimadzu FT IR-4200
Fig. 3. ${ }^{1} \mathrm{H}-{ }^{1} \mathrm{H}$ COSY and $\mathrm{HMBC}$ experiments of pyridomacrolidin (2).

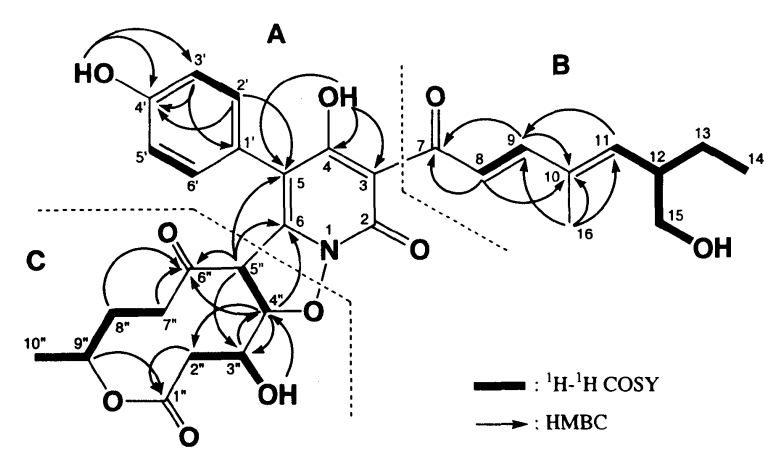

spectrophotometer and Shimadzu UV-3100 spectrophotometer, respectively. Optical rotation was measured in $\mathrm{MeOH}$ on a JASCO DIP-370 digital polarimeter.

\section{References}

1) RoBerts, D. W.: Toxins of entomopathogenic fungi. In Microbial Control of Pests and Plant Diseases 1970 1980. Ed., H. D. Burges, pp. $441 \sim 464$, Academic Press, London, New York, 1981

2) Fujita, T.; K. Inoue, S. Yамамото, T. Iкumoto, S. Sasaki, R. Toyama, K. Chiba, Y. Hoshino \& $\mathrm{T}$. Окимото: Fungal metabolites. Part 11. A potent immunosuppressive activity found in Isaria sinclairii metabolite. J. Antibiotics 47: 208 215, 1994

3) Iijima, M.; T. Masuda, H. Nakamura, H. Naganawa, S. Kurasawa, Y. OKami, M. Ishizuka, T. TAKeuchi \& Y. IITAKA: Metacytofilin, a novel immunomodulator produced by Metarhizium sp. TA2759. J. Antibiotics 45: $1553 \sim 1556,1992$

4) Takahashi, S.; N. KaKinuma, K. UChida, R. HaShIMOTO, T. Yanagisawa \& A. NaKagawa: Pyridovericin and pyridomacrolidin: novel metabolites from entomopathogenic fungi, Beauveria bassiana. J. Antibiotics 51: 596 598, 1998

5) WAt, C. K.; A. G. MCInNes, D. G. Smith, J. L. C. WRight \& L. C. VINING: The yellow pigments of Beauveria species. Structures of tenellin and bassianin. Can. J. Chem. 55: 4090 4098, 1977

6) Matsumoto, M. \& H. Minato: Structure of ilicicolin $\mathrm{H}$, an antifungal antibiotic. Tetrahedron Lett.: $3827 \sim 3830$, 1976 